zweiten einen mehrwöchentlichen Krankenhausaufenthalt notwendig. Ihre Erklärung ist nicht so ganz leicht. Wir sind auf Vermutungen angewiesen. Aber sollte es ganz von der Hand zu weisen sein, dass längs der Fraktur Infektionserreger von der Nase oder der Schleimhaut der Nebenhöhle zu den Hirnhäuten vorgedrungen sind und eine Meningitis hervorgerufen haben? Diese Annahme zu machen erscheint mir jedenfalls berechtigter, als jeglichen Zusammenhang zwischen der Operation und den nachfolgenden Krankheitserscheinungen $\mathrm{zu}$ leugnen, wie es in dem der Krankengeschichte des Krankenhauses angefügten Résumé geschieht.

Beide Fälle bilden einen neuen interessanten Beitrag auf dem Gebiete der in letzter Zeit immer mehr erforschten Beziehungen der Erkrankungen der Nase zu denen des Auges. Wir sehen nach einem von den Rhinologen oft vorgenommenen, wohl bis dahin als gefahrlos angesehenen Eingriff schwere bleibende Sehstörungen eintreten, wie sie in gleicher Weise bisher hauptsächlich nach Schädelverletzungen in Schläfen- und Supraorbitalgegend beobachtet worden sind. Wir müssen nach Analogic mit diesen Fällen auch in den unserigen eine Verletzung der Sehnerven durch Fraktur ihrer Wandung annehmen. Und unsere Schilderung diarf wohl in die Mahnung für die Nasenärzte ausklingen, bei der Operation weit nach hinten und oben sich erstreckender Septumdeviationen Zurïckhaltung $\mathrm{zu}$ ïben, damit nicht die Kasuistik auf diesem Gebiete noch vermehrt werde.

\title{
Literatur.
}

1. Onodi, A., Die Sehstörungen und Erblindung nasalen Urrsprungs, bedingt durch die Erkrankungen der hinteren Nasenhühlen. Zeitschr. f. Augenheilk. I3d. XII. S. 23.

2. Derselbe, Die Aetiologie der kontralateralen Sehstörung und Frblindung nasalen Ursprungs. Bericht über dic 33. Versammlung der Ophthalmologischen Gesellschaft. Heidelberg 1906. S. 15.3.

3. Jerselbe, Jie Nebenhöhlen der Nase. Atlas. Wien 1905.

4. Berlin, Die Verletzungen der Orbita. In Graefe-Saemisch' Handbuch f. Augenheilk. 1. Aufl. 13d. VI. S. $615 \mathrm{u}$. ff.

5. Freudenthal, Archive international de laryngologie. 1905. I3d. II. S. 761.

6. Wilbrand-Saenger, Die Neurologie des Auges. Bd. III. 2. Hälfte. S. 752.

VI.

\section{Über Übertragung von Hautschollen nach der Enukleation bei eingeengtem Bindehautsack.}

Von

\author{
HERMANN K U H T-Bonn.
}

Tritt die Notwendigkeit einer Enukleation bei wesentlich eingeengtem Bindehautsacke (bei inveteriertem Trachom, Verbrennungs- oder Verätzungsnarben etc.) an uns heran, so resul- 
tiert bekanntlich zumeist eine derartige Verkleinerung des Konjunktivalsackes, dass das Tragen eines nur annähernd entsprechend grossen künstlichen Auges zur Unmöglichkeit wird, auch die Beweglichkeit der Prothese nur gar zu viel zu wünschen übrig lässt. Nach mehrfachen Versuchen, für diese Fälle eine Abhïlfe zu schaffen, bin ich zu folgendem, wie mir scheint, überaus leichten und naheliegenden Verfahren gelangt.

Die Enukleation wird unter Fixierung der Sehnen der geraden Augenmuskeln an die Konjunktiva ausgeführt, wodurch, wie Heinrich Schmidt dargetan, die Beweglichkeit der späteren Prothese in hohem Masse gewinnt. Meine Technik der Sehnenfixierung an die Bindehaut ist eine sehr vereinfachte und leichte. Nachdem im unteren äusseren Quadranten des Bulbus ein 5 bis $6 \mathrm{~mm}$ langer, zum Hornhautrande paralleler Einschnitt in den Limbus gemacht und hierauf mit der Blömerschen Pinzette die vordere Insertion der T'enonschen Binde gefasst und durchtrennt worden ist, führe ich den ausgehöhlten Schiclhaken') unter die Sehne des MIusc. rect. externus und leite durch sie samt darüber liegender Bindehaut, - welche beide mittelst des Schielhakens leicht gespannt werden, - eine kräftige Fadenschlinge, in der Art, dass die halbkreisförmige Nadel durch Konjunktiva, Subkonjunktiva und Sehne gestochen, in der Aushöhlung des Hakens 2 bis $3 \mathrm{~mm}$ unter der Sehne rorgeschoben und nun ron innen nach aussen durch die genannten Gewebe wieder durchgestossen wird. Feste Knotung der Fadenschlinge. Es folgt die Durchtrennung der Bindehaut am äusseren Hornhautrande und der Insertionsleiste der Sehne des Externus sowie der Anheftungsstelle der Tenonschen Binde im obcren äusseren Bulbusquadranten. Hierauf wird mit einem entgegengesetzt abgebogenen ausgehöhlten Schielhaken in derselben Weise die Sehne des Rect. inferior, später des internus und superiors, aufgeladen und mit je einem Faden an die Bindehaut geknotet. Die weitere Enukleation schliesst sich in der gewohnten Weise an. Nach Stillung der etwaigen Blutung übertrage ich nunmehr eine von der Innenfläche des Oberarmes oder Oberschenkels entnommene T'hierschsche Hautscholle auf dic durch die Entfernung des Augapfels gesetzte Wundstelle. Letztere ist in solchen Fällen gewöhnlich recht gross, da dic narbig entartete Bindehaut sich naturgemäss sehr stark nach dem Lidknorpel und den Augenwinkeln zu retrahiert. Es bleibt zweckmässig, ein recht grosses und annähernd rund gestaltetes Hautstïck zu transplantieren und $\mathrm{zu}$ dem Behufe seine Ränder so weit wie angängig unter die Bindehaut zu schieben. Um die Hautscholle in dauerndem festen Kontakte mit der Wundfläche zu erhalten, lege ich entweder eine aus Holz gefertigte Kugel von etwa 18 bis $20 \mathrm{~mm}$ Durchmesser, bezw. ein Holzei (lange Achse 20 bis 2.2 mm, kurze Achse 14 his $16 \mathrm{~mm}$ ) oder

1) Vgl. die Abbildungen im VI. Bd. dieser \%eitschrift. pag. \&6f. Die Schichaken werden verfertigt rom Instrumentenfabrikant Palm in Königsberg i. l'r.. Altstiidtische J,anggasse. 
einen ähnlich gestalteten Pfropf aus hydrophilem Verbandstoffe in den Bindehautsack, schliesse darüber die Lidspalte durch einen Faden, der die Haut des Randes vom oberen und unteren Ijide in einer Ausdehnung von 2 bis $3 \mathrm{~mm}$ gefasst hatte, und lege einen Druckverband an. Dieser hat möglichst lange, jedenfalls 3 bis 4 Tage, liegen zu bleiben. Die Anheilung erfolgt ohne jede Reaktion. Selbstredend tritt nach und nach eine mehr oder weniger beträchtliche Schrumpfung des übertragenen Hautstückes ein, immerhin aber erwächst eine so beträchtliche Erweiterung des Bindehautsackes, dass zumeist ein befriedigend grosses künstliches Auge getragen werden kann. Auch die Beweglichkeit der Prothese ist gemeinhin eine recht gute.

Zum Schluss bemerke ich noch, dass sich die Fixierung der Sehnenenden der graden Muskeln an die Bindehaut auf die angegebene Art mit Benutzung der ausgehöhlten Schielhaken in allen Fällen bestens bewährte. Jedenfalls sollte sie bei nötig werdenden Enukleationen im Interesse einer befriedigenden Bcweglichkeit der Prothese, zumal ihre Ausführung weder Schwierigkeit noch nennenswerten Zeitverlust verursacht, beachtet werden . Ebenso warm aber möchte ich auch die mühelose Vergrösserung des Bindehautsackes bei zur Schrumpfung neigenden Erkrankungen der Konjunktiva oder bei schon vorhandenen Einengungen durch t'bertragung einer grossen Hautscholle auf die Enukleationswunde empfehlen.

\section{Berichte und Referate.}

\section{Bericht über die deutsche ophthalmologische Literatur. \\ Physiologie des Gesichtssinns. \\ I. und II. Semester 1906.

\author{
Referent: Privatdozent 1)r. GEORG FR. NICOLAI, \\ Assistent am physiologischen Institut in Berlin.
}

(Schluss.)

Blessing (45) bespricht die physiologischen und pathologischen Wirkungen monochromatischer Lichtarten anf das Auge, ausserdem deren therapeutische Verwendung.

Einen ganz neuen Coesichtspunkt für die Beurteilung der Lichtwirkungen bieten die Untersuchungen von Jacupes Loeb (46). Schon frïher hatte er gezeigt, dass man imstande ist, durch 'Temperatureinflüsse und durch Konzentrationsänderungen bei manchen Tieren Heliotropismus (also die Fähigkeit. Licht in irgend einer Weise zu per\%ipieren) willkürlich hervorzurufen. In dieser Arbeit zeigt er nun, dass dies auch durch /usat\% geringer Säuremengen gelingt. Vor allen Dingen aber 